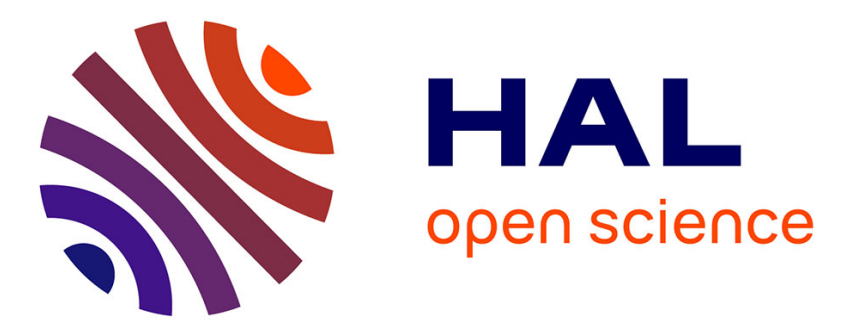

\title{
Stochastic Subspace-Based Damage Detection with Uncertainty in the Reference Null Space
}

\author{
Eva Viefhues, Michael Döhler, Falk Hille, Laurent Mevel
}

\section{To cite this version:}

Eva Viefhues, Michael Döhler, Falk Hille, Laurent Mevel. Stochastic Subspace-Based Damage Detection with Uncertainty in the Reference Null Space. IWSHM - 11th International Workshop on Structural Health Monitoring, Sep 2017, Stanford, United States. 10.12783/shm2017/13963 . hal01589540

\author{
HAL Id: hal-01589540 \\ https://hal.inria.fr/hal-01589540
}

Submitted on 18 Sep 2017

HAL is a multi-disciplinary open access archive for the deposit and dissemination of scientific research documents, whether they are published or not. The documents may come from teaching and research institutions in France or abroad, or from public or private research centers.
L'archive ouverte pluridisciplinaire HAL, est destinée au dépôt et à la diffusion de documents scientifiques de niveau recherche, publiés ou non, émanant des établissements d'enseignement et de recherche français ou étrangers, des laboratoires publics ou privés. 
Title: Stochastic Subspace-Based Damage Detection with Uncertainty in the Reference Null Space

\author{
Authors : Eva VIEFHUES ${ }^{1}$ \\ Michael DÖHLER ${ }^{2,3}$ \\ Falk HILLE ${ }^{1}$ \\ Laurent MEVEL ${ }^{2,3}$
}




\begin{abstract}
This paper deals with uncertainty considerations in damage diagnosis using the stochastic subspace-based damage detection technique. With this method, a model is estimated from data in a (healthy) reference state and confronted to measurement data from the possibly damaged state in a hypothesis test. Previously, only the uncertainty related to the measurement data was considered in this test, whereas the uncertainty in the estimation of the reference model has not been considered. We derive a new test framework, which takes into account both the uncertainties in the estimation of the reference model as well as the uncertainties related to the measurement data. Perturbation theory is applied to obtain the relevant covariances. In a numerical study the effect of the new computation is shown, when the reference model is estimated with different accuracies, and the performance of the hypothesis tests is evaluated for small damages. Using the derived covariance scheme increases the probability of detection when the reference model estimate is subject to high uncertainty, leading to a more reliable test.
\end{abstract}

\title{
INTRODUCTION
}

Vibration based monitoring, as it is used for structural health monitoring (SHM), takes advantage of the fact that the dynamical behavior of a structure is affected by damages [1]. Stochastic subspace identification (SSI) methods has been proven useful for the identification of civil and mechanical structures under ambient excitation, as those approaches provide output-only algorithms and particular information about the excitation is not needed [2]. Based on subspace properties of SSI, the stochastic subspacebased damage detection (SSDD) technique [3-5] sets up a statistical framework, where a reference model is estimated in a (healthy) reference state of the structure. It is then confronted to measurements of the current system, without repeating the system identification step in the testing states.

\footnotetext{
${ }^{1}$ Federal Institute for Materials Research and Testing (BAM), Division 7.2 Buildings and Structures, Berlin, Germany. Email: eva.viefhues@bam.de

${ }^{2}$ Inria, I4S, Campus de Beaulieu, Rennes, France.

${ }^{3}$ IFSTTAR, COSYS, SII, Bouguenais, France
} 
In detail, the null space of the Hankel matrix of output covariances is estimated in the reference state, which is related to the reference model. Together with measurement data of the current system, a residual vector is generated based on the reference null space and the estimated output covariances of the current system. The residual is statistically evaluated in a hypothesis test to decide if the system has changed. For this decision, the computed test statistic is compared to a threshold in the monitored state. Various works are published, showing the successful application of the method to numerical and real case studies, e.g. [6].

The test performance is highly dependent on the covariance estimate of the considered residual, which is a related to the stochastic nature of the measured signals. Currently, the hypothesis tests take into account the uncertainties of the measured data for the residual covariance [7], but uncertainties related to the reference null space are not considered. The current theory is thus limited to ideal cases, where the reference model is well known. However, the reference null space is also estimated from data in practice and thus subject to uncertainties. Consequently, these uncertainties should also be taken into account for the statistical evaluation of the residual.

In this paper we show how to obtain a residual covariance taking into account uncertainties of the data and also of the reference null space by using perturbation propagation theory. The effect of the new residual covariance on the test performance is shown and discussed in a numerical study.

In the following chapter we recall the theoretical background of the SSDD method. Then we present the derivation of the residual covariance taking into account uncertainties in the reference null space, before applying the new approach in a numerical study.

\section{SUBSPACE-BASED DAMAGE DIAGNOSIS}

\section{Stochastic subspace-based residual}

The vibration behavior of a mechanical structure can be described by a linear timeinvariant dynamical system

$$
\mathbf{M} \ddot{z}(t)+\mathbf{C} \dot{z}(t)+\mathbf{K} z(t)=v_{F}(t),
$$

where $t$ denotes continuous time, $M, C$ and $K \in \mathbb{R}^{m \times m}$ are the mass, damping and stiffness matrices. Vector $z(t) \in \mathbb{R}^{m}$ contains the displacements of the $m$ degrees of freedom of the structure. $v_{F}(t)$ is an external force, which is usually unknown for civil structures and modeled as white noise. Observing the structural system in (1) with a set of $r$ acceleration sensors we get the measurements

$$
y(t)=\mathbf{L} \ddot{z}(t)+e(t)
$$

where $y \in \mathbb{R}^{r}$ is the measurement vector, matrix $L \in \mathbb{R}^{r \times m}$ contains the sensor locations, and $e$ is the measurement noise.

Measurements are recorded at discrete time instants $k$ with a sampling rate $\tau$. The stochastic discrete time state-space model corresponding to (1) is considered, namely

$$
\left\{\begin{array}{l}
x_{k+1}=A x_{k}+w_{k} \\
y_{k}=C x_{k}+v_{k}
\end{array}\right.
$$


where $x \in \mathbb{R}^{n}$ denotes the state, and the measured output is $y \in \mathbb{R}^{r}$. The system matrices $A$ and $C$ denote the state transition matrix with dimension $m \times m$ and the observation matrix with dimension $r \times m$, respectively. The state noise $w_{k}$ and output noise $v_{k}$ are assumed to be white noise, possibly correlated.

For damage diagnosis, it is checked if data from the current state of the structure fits to a reference model or not. This is done by evaluating a residual vector. The residual is based on the covariance-driven output-only subspace method, using the fact that the system matrices $A$ and $C$ are linked to the block Hankel matrix

$$
\mathcal{H} \stackrel{\text { def }}{=}\left[\begin{array}{cccc}
R_{1} & R_{2} & \ldots & R_{q} \\
R_{2} & R_{3} & \ldots & R_{q+1} \\
\vdots & \vdots & \ddots & \vdots \\
R_{p+1} & R_{p+2} & \ldots & R_{q+p}
\end{array}\right]=\operatorname{Hank}\left(R_{i}\right)
$$

containing the output covariance $R_{i}=\mathbf{E}\left(y_{k} y_{k-i}^{T}\right)=C A^{i-1} G$ with $G=\mathbf{E}\left(x_{k+1} y_{k}^{T}\right)$, as it is described in detail in [4]. Let $S$ be the left null space matrix of $\mathcal{H}$ in the reference state, such that $S^{T} \mathcal{H}_{\text {ref }}=0$ holds. $S$ is referred to as the reference null space, which is related to the reference model. Let $\hat{\mathcal{H}}$ be an estimate of $\mathcal{H}$ in the current state of the structure, which is estimated from $\hat{R}_{i}=\frac{1}{N} \sum_{k=1}^{N} y_{k} y_{k-i}^{T}$ using $N$ measured outputs $\left\{y_{k}\right\}_{k=1, \ldots, N^{*}}$. Based on the null space property of $S$ in the reference state, the residual vector $\zeta$ is defined as

$$
\zeta=\sqrt{N} \operatorname{vec}\left(S^{T} \hat{\mathcal{H}}\right)
$$

where $\operatorname{vec}(\cdot)$ denotes the column stacking vectorization operator. The expected value of $\zeta$ deviates from 0 , if the system deviates from the reference state and thus the expected value of $\hat{\mathcal{H}}$ changes compared to $\mathcal{H}_{\text {ref }}$. It has been shown that the residual vector is asymptotically Gaussian distributed (for large $N$ )

$$
\zeta \longrightarrow \begin{cases}\mathcal{N}\left(0, \Sigma_{\zeta}\right) & \text { undamaged state } \\ \mathcal{N}\left(\mathcal{J}_{\zeta, \theta} \delta, \Sigma_{\zeta}\right) & \text { damaged state }\end{cases}
$$

with zero mean in the reference state and non-zero mean in the damaged state. In here, $\mathcal{J}_{\zeta, \theta}$ is the sensitivity of the residual in the reference state with respect to a reference system parameter $\theta=\theta_{0}$ (e.g. containing the collection of modal or structural parameters), $\delta$ is unknown but fixed, and $\Sigma_{\zeta}$ is the residual covariance. Note that both the residual sensitivity and the covariance are computed in the reference state, details are given in [4].

\section{Hypothesis testing}

The evaluation of changes in the residual and thus in its distribution properties is done by means of hypothesis tests. Generalized likelihood ratio (GLR) tests are used, as proposed e.g. in $[4,5,8]$. They give the information whether the measured output corresponds more likely to a system with $\theta=\theta_{0}$ or to a system with a changed system parameter. The hypotheses can be written as

$$
\begin{array}{ll}
\text { for the reference system } & \mathbf{H}_{0}: \theta=\theta_{0} \\
\text { for the damaged system } & \mathbf{H}_{1}: \theta=\theta_{0}+\delta / \sqrt{N},
\end{array}
$$


and the hypothesis test writes

$$
t=\zeta^{T} \Sigma_{\zeta}^{-1} \mathcal{J}_{\zeta, \theta}\left(\mathcal{J}_{\zeta, \theta}^{T} \Sigma_{\zeta}^{-1} \mathcal{J}_{\zeta, \theta}\right)^{-1} \mathcal{J}_{\zeta, \theta}^{T} \Sigma_{\zeta}^{-1} \zeta
$$

The test statistic $t$ is asymptotically $\chi^{2}$ distributed, having $d=\operatorname{rank}\left(\mathcal{J}_{\zeta, \theta}\right)$ degrees of freedom. It is central in the reference state, and non-central in the damaged state with non-centrality parameter $\lambda=\delta^{T} \mathcal{J}_{\zeta, \theta}^{T} \Sigma_{\zeta}^{-1} \mathcal{J}_{\zeta, \theta} \delta$.

The diagnosis of the system's health state finally is made by comparing the test statistic $t$ to a threshold, which has to be computed in a training phase in the undamaged reference state for a given type I error.

\section{RESIDUAL COVARIANCE WITH UNCERTAINTY IN REFERENCE MODEL}

The performance of the hypothesis test in (8) is highly dependent on the residual covariance $\Sigma_{\zeta}$. Up to now, only the covariance $\Sigma_{\mathcal{H}}=\lim \operatorname{cov}(\sqrt{N} \operatorname{vec}(\hat{\mathcal{H}}))$ related to the data in $\hat{\mathcal{H}}$ itself is considered in its computation. However, the null space matrix $S$ describing our reference model in the residual is usually not perfectly known in practice, but only an estimate $\hat{S}$ of $S$ is obtained from measurements in the reference state and thus afflicted with uncertainties. Hence, its covariance should be also be considered in the computation of the residual covariance, which is described in the following. First, the residual covariance is related to both covariance contributions regarding the estimated reference null space $\hat{S}$ and the data in the Hankel matrix $\hat{\mathcal{H}}$ based on the propagation of first-order perturbations. Second, the covariance related to $\hat{S}$ is described in detail.

\section{Perturbation Approach for Covariance Computation}

Let $\hat{Y}=f(\hat{X})$ be a vector valued function of some estimate $\hat{X}$ of $X$. A first order Taylor approximation yields $f(\hat{X}) \approx f(X)+\mathcal{J}_{Y, X}(\hat{X}-X)$, or in delta notation describing first-order perturbations, $\Delta Y \approx \mathcal{J}_{Y, X} \Delta X$, where $\mathcal{J}_{Y, X}$ is the derivative of $f$, and $\Delta X=\hat{X}-X$ for $\hat{X}$ close to $X$. Then, the covariance of the function of the estimate can be expressed as $\operatorname{cov}(\hat{Y}) \approx \mathcal{J}_{Y, X} \operatorname{cov}(\hat{X}) \mathcal{J}_{Y, X}^{T}$.

For the proposed covariance computation the residual function in Equation (5) is considered as a function of two multivariate random variables $\hat{S}$ and $\hat{\mathcal{H}}$, instead of a function of only one variable $\hat{\mathcal{H}}$. A first-order perturbation of the residual then yields

$$
\begin{aligned}
\Delta \zeta & =\operatorname{vec}\left(\sqrt{N} \Delta S^{T} \hat{\mathcal{H}}\right)+\operatorname{vec}\left(\sqrt{N} \hat{S}^{T} \Delta \mathcal{H}\right) \\
& =\underbrace{\left(\hat{\mathcal{H}}^{T} \otimes I\right) \mathcal{P}_{(p+1) r \times q r-n}}_{\mathcal{J}_{\zeta, S}} \operatorname{vec}(\sqrt{N} \Delta S)+\underbrace{\left(I \otimes \hat{S}^{T}\right)}_{\mathcal{J}_{\zeta, \mathcal{H}}} \operatorname{vec}(\sqrt{N} \Delta \mathcal{H}),
\end{aligned}
$$

using the relation $\operatorname{vec}(A X B)=\left(B^{T} \otimes A\right) \operatorname{vec}(X)$, where $\otimes$ is the Kronecker product, and $\mathcal{P}_{a, b}$ is a permutation matrix such that $\operatorname{vec}\left(X^{T}\right)=\mathcal{P}_{a, b} \operatorname{vec}(X)$ for a matrix $X \in \mathbb{R}^{a \times b}$ [7]. It holds $S \in \mathbb{R}^{(p+1) r \times(p+1) r-n}$ and $\mathcal{H} \in \mathbb{R}^{(p+1) r \times q r}$, where $r$ is the number of sensors, and $n$ denotes the system order.

Let $\hat{S}$ be obtained from a Hankel matrix $\hat{\mathcal{H}}^{0}$, using the singular value decomposition (SVD)

$$
\hat{\mathcal{H}}^{0}=\left[\begin{array}{ll}
U_{1} & U_{2}
\end{array}\right]\left[\begin{array}{cc}
D_{1} & 0 \\
0 & D_{2}
\end{array}\right]\left[\begin{array}{l}
V_{1}^{T} \\
V_{2}^{T}
\end{array}\right]
$$


with $\hat{S}=U_{2}$. Then, a perturbation of $S$ yields the relation $\operatorname{vec}(\Delta S)=\mathcal{J}_{S, \mathcal{H}^{0}} \operatorname{vec}\left(\Delta \mathcal{H}^{0}\right)$, where the sensitivity $\mathcal{J}_{S, \mathcal{H}^{0}}$ will be elaborated in the subsequent section. Finally,

$$
\begin{aligned}
\Delta \zeta & =\mathcal{J}_{\zeta, S} \mathcal{J}_{S, \mathcal{H}^{0}} \operatorname{vec}\left(\sqrt{N} \Delta \mathcal{H}^{0}\right)+\mathcal{J}_{\zeta, \mathcal{H}} \operatorname{vec}(\sqrt{N} \Delta \mathcal{H}) \\
& =\underbrace{\left[\begin{array}{ll}
\mathcal{J}_{\zeta, S} \mathcal{J}_{S, \mathcal{H}^{0}} & \mathcal{J}_{\zeta, \mathcal{H}}
\end{array}\right]}_{\mathcal{A}}\left[\begin{array}{c}
\operatorname{vec}\left(\sqrt{N} \Delta \mathcal{H}^{0}\right) \\
\operatorname{vec}(\sqrt{N} \Delta \mathcal{H})
\end{array}\right]
\end{aligned}
$$

For the calculation of $\Sigma_{\zeta}$, covariance estimates of $\Sigma_{\mathcal{H}}=\lim \operatorname{cov}(\operatorname{vec}(\sqrt{N} \hat{\mathcal{H}}))$ and of $\Sigma_{\mathcal{H}^{0}}=\lim \operatorname{cov}\left(\operatorname{vec}\left(\sqrt{N} \hat{\mathcal{H}}^{0}\right)\right)$ are used, which can be obtained easily from data. The procedure is e.g. described in [9]. Matrix $\hat{\mathcal{H}}^{0}$ and the corresponding null space $\hat{S}$ are estimates obtained in the reference state of the system, and $\hat{\mathcal{H}}$ is an estimate obtained in the current system for the damage detection test. Thus both matrices $\hat{\mathcal{H}}^{0}$ and $\hat{\mathcal{H}}$ are obtained from different datasets and are statistically independent. Then, the residual covariance follows from (11) as

$$
\begin{aligned}
\Sigma_{\zeta} & =\mathcal{A}\left[\begin{array}{cc}
\Sigma_{\mathcal{H}^{0}} & 0 \\
0 & \Sigma_{\mathcal{H}}
\end{array}\right] \mathcal{A}^{T} \\
& =\mathcal{J}_{\zeta, S} \mathcal{J}_{S, \mathcal{H}^{0}} \Sigma_{\mathcal{H}^{0}} \mathcal{J}_{S, \mathcal{H}^{0}}^{T} \mathcal{J}_{\zeta, S}^{T}+\mathcal{J}_{\zeta, \mathcal{H}} \Sigma_{\mathcal{H}} \mathcal{J}_{\zeta, \mathcal{H}}^{T}
\end{aligned}
$$

In fact, both covariances $\Sigma_{\mathcal{H}^{0}}$ and $\Sigma_{\mathcal{H}}$ can be estimated in the reference state of the system thanks to the small change hypothesis [10], and it holds $\Sigma_{\mathcal{H}^{0}}=\Sigma_{\mathcal{H}}$ in (12).

\section{Sensitivities of the Reference Null Space}

Now, the required sensitivity $\mathcal{J}_{S, \mathcal{H}^{0}}$ of the null space matrix is derived for Equation (12). Consider $\hat{S}=U_{2}$ is calculated using the SVD in (10), thus the perturbation of $\mathcal{H}^{0}$ is propagated to the left null space vectors within the SVD.

Let $U_{1}$ and $U_{2}$ be the the column space and the left null space of $\hat{\mathcal{H}}^{0}, U_{1} \in \mathbb{R}^{h \times n}$ and $U_{2} \in \mathbb{R}^{h \times s}$, where $n$ is the number of non-zero singular values, $s=(p+1) r-n$ and $h=(p+1) r$. For the perturbation propagation from $\hat{\mathcal{H}}^{0}$ to its column space $U_{1}$ it holds [11]

$$
\Delta U_{1}=U_{1} R+U_{2} U_{2}^{T} \Delta \mathcal{H}^{0} V_{1} \Delta_{1}^{-1}
$$

where $R$ is a matrix that will be canceled in the following, and the expected values of all singular values in $\Delta_{1}$ are distinct from zero. In the vectorized form it follows

$$
\operatorname{vec}\left(\Delta U_{1}\right)=\left(I_{n} \otimes U_{1}\right) \operatorname{vec}(R)+\left(\left(V_{1} \Sigma_{1}^{-1}\right)^{T} \otimes U_{2} U_{2}^{T}\right) \operatorname{vec}\left(\Delta \mathcal{H}^{0}\right)
$$

This perturbation is now propagated to the left null space $U_{2}$. From $U_{1}^{T} U_{2}=0$ it follows

$$
\begin{aligned}
& I_{n} \Delta U_{1}^{T} U_{2}+U_{1}^{T} \Delta U_{2} I_{s}=0 \\
& \Rightarrow\left(I_{s} \otimes U_{1}^{T}\right) \operatorname{vec}\left(\Delta U_{2}\right)=-\left(U_{2}^{T} \otimes I_{n}\right) \mathcal{P}_{h, n} \operatorname{vec}\left(\Delta U_{1}\right) .
\end{aligned}
$$

Considering $U_{2}^{T} U_{2}=I$ and thus $\Delta\left(U_{2}^{T} U_{2}\right)=0$, it follows $\Delta U_{2}^{T} U_{2}+U_{2}^{T} \Delta U_{2}=0$ and

$$
\mathcal{P}_{s, s}\left(I_{s} \otimes U_{2}^{T}\right) \operatorname{vec}\left(\Delta U_{2}\right)+\left(I_{s} \otimes U_{2}^{T}\right) \operatorname{vec}\left(\Delta U_{2}\right)=0
$$


Thus, with (15) and (16), a perturbation of the left null space satisfies

$$
\left[\begin{array}{ll}
I_{s n} & \\
& \mathcal{P}_{s, s}+I_{s^{2}}
\end{array}\right]\left[\begin{array}{l}
I_{s} \otimes U_{1}^{T} \\
I_{s} \otimes U_{2}^{T}
\end{array}\right] \operatorname{vec}\left(\Delta U_{2}\right)=\left[\begin{array}{c}
-\left(U_{2}^{T} \otimes I_{n}\right) \mathcal{P}_{h, n} \\
0
\end{array}\right] \operatorname{vec}\left(\Delta U_{1}\right) .
$$

As the first matrix on the left handside is in general not invertible, consider the particular solution

$$
\begin{aligned}
\operatorname{vec}\left(\Delta U_{2}\right) & =-\left(I_{s} \otimes U_{1}\right)\left(U_{2}^{T} \otimes I_{n}\right) \mathcal{P}_{h, n} \operatorname{vec}\left(\Delta U_{1}\right) \\
& =-\mathcal{P}_{s, h}\left(U_{1} \otimes U_{2}^{T}\right) \operatorname{vec}\left(\Delta U_{1}\right) .
\end{aligned}
$$

We now have the perturbation propagation from a matrix to its left null subspace by plugging (14) into (18):

$$
\begin{aligned}
\operatorname{vec}\left(\Delta U_{2}\right) & =-\mathcal{P}_{s, h}\left(U_{1} \otimes U_{2}^{T}\right)\left[\left(I_{n} \otimes U_{1}\right) \operatorname{vec}(R)+\left(\left(V_{1} D_{1}^{-1}\right)^{T} \otimes U_{2} U_{2}^{T}\right) \operatorname{vec}\left(\Delta \mathcal{H}^{0}\right)\right] \\
& =\underbrace{-\mathcal{P}_{s, h}\left(U_{1} D_{1}^{-1} V_{1}^{T} \otimes I_{s}\right)\left(I_{q r} \otimes U_{2}^{T}\right)}_{\mathcal{J}_{U_{2}, \mathcal{H}^{0}}} \operatorname{vec}\left(\Delta \mathcal{H}^{0}\right)
\end{aligned}
$$

With $\hat{S}=U_{2}$, the product $\mathcal{J}_{\zeta, \mathcal{H}^{0}}=\mathcal{J}_{\zeta, S} \mathcal{J}_{S, \mathcal{H}^{0}}$ in (12) melts down to

$$
\begin{aligned}
\mathcal{J}_{\zeta, \mathcal{H}^{0}} & =\left(\hat{\mathcal{H}}^{T} \otimes I_{s}\right) \mathcal{P}_{h, s}\left(-\mathcal{P}_{s, h}\right)\left(U_{1} D_{1}^{-1} V_{1}^{T} \otimes I_{s}\right)\left(I_{q r} \otimes \hat{S}^{T}\right) \\
& =-\left(\hat{\mathcal{H}}^{T} U_{1} D_{1}^{-1} V_{1}^{T} \otimes I_{s}\right)\left(I_{q r} \otimes \hat{S}^{T}\right)=-V_{1} V_{1}^{T} \otimes \hat{S}^{T} .
\end{aligned}
$$

This holds since $\hat{\mathcal{H}}$ and $\hat{\mathcal{H}}^{0}$ are consistent estimates of the same matrix for the covariance computation in the reference state of the system, and thus their subspaces are equal.

\section{APPLICATION}

In a numerical study the effect of the new covariance comparison is evaluated by comparing results to the previous computation method that does not take into account uncertainties in the reference null space. We consider a 8-mass-spring-damper with $1.5 \%$ damage in the damaged state at element 3 . The null space $\hat{S}$ and the residual sensitivity and covariance are computed in the reference state. The considered system parameter $\theta$ is chosen to be the stiffnesses of the 8 springs. Thus the $\chi^{2}$-test has 8 degrees of freedom, and the expected value of the test statistic in the reference state is 8 .

Figure 1 shows the distribution of the test statistic in Monte Carlo simulations. It is shown in healthy and damaged states using the previous and the new covariance computation. The null space $\hat{S}$ and the covariances are computed on $N=50000$ samples in the reference state. In this case it can be seen that the mean of the test statistic deviates significantly from its theoretical value of 8 when not considering the uncertainty on $\hat{S}$ in the previous computation. This is due to the fact that the reference null space may not be perfectly estimated, and the previous algorithm does not consider the related uncertainty in the hypothesis test. Nevertheless, the distribution in the damaged state is shifted toward higher $\chi^{2}$-values, and damage can be detected in most cases when choosing an empirical threshold in the reference state. Choosing a threshold from the theoretical distribution in the reference state is not possible in this case, since it only leads to false alarms. 


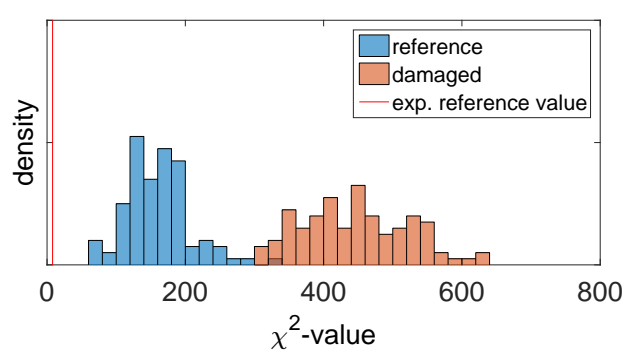

(a) Previous covariance computation

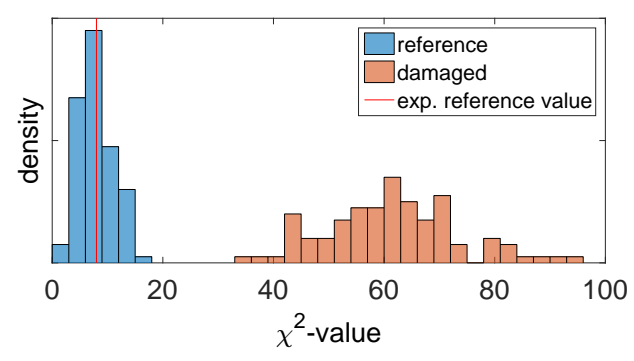

(b) New covariance computation

Figure 1. Histograms of test statistic for previous (a) and new (b) covariance computation.

With the new residual covariance, the theoretical mean of the test statistic in the reference state is well obtained, as the uncertainties related to the reference null space are taken into account. Moreover, the distribution in the damaged state is better separated from the distribution in the reference state, leading to a better probability of detection. So both theoretical or empirical thresholds will not give false alarms in the undamaged state, and damages can be better detected.

In Figure $2 \mathrm{a}$ the mean of the test statistic is shown for the previous and the new covariance computation in the reference state. Different data lengths for computation of $\hat{S}$ and the covariances are considered. It can be seen that the previous computation method needs more data to reach the theoretical test value, whereas the convergence behavior with the new covariance scheme is much better. If enough data is available, the previous computation method gives also proper results. This is due to the fact, that the estimation of the reference null space is more precise with more data, and then its contribution to the residual covariance decreases. However, the new covariance computation should be preferred when only few measurements are available in the reference state.

To illustrate both algorithms' performance in the damaged state, the ratio the test value means between the damaged and the undamaged state is shown in Figure $2 \mathrm{~b}$. Considering the distribution plots in Figure 1, the ratio measures how far the mean shifts in the tested state. The tests using the new covariance perform better for less data. The impact of considering the covariance of $\hat{S}$ decreases when more data is available.

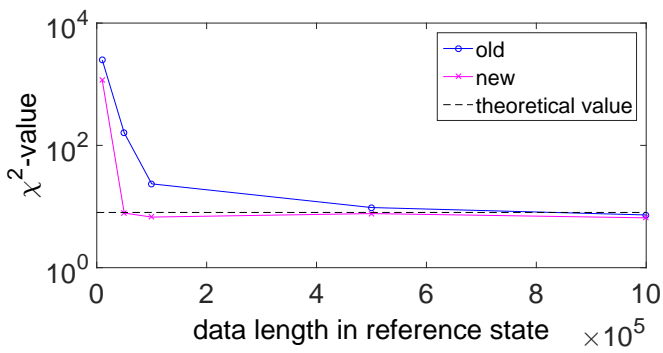

(a) Mean of test values in reference state

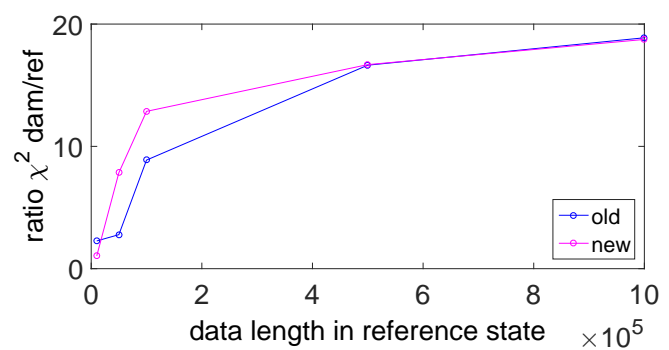

(b) Ratio of test value means damaged / reference state

Figure 2. Mean of test statistic (a) and test performance (b) for previous and new covariance computation in dependence of data length in reference state. 


\section{CONCLUDING REMARKS}

A new computation method has been provided for the residual covariance in the subspace-based damage detection method. On top of the uncertainties related to measurement data that is tested for damage, the new computation takes also into account the uncertainties related to the reference model properly. As a result the computed test values in the reference state are very close to the theoretically expected values and the test performance is more reliable in the damaged state. Numerical results show that the uncertainties related to the reference model should not be neglected, especially when few data is available in the reference state.

\section{ACKNOWLEDGMENT}

The financial support from DAAD/German Federal Ministry of Education and Research and Campus France in the PROCOPE project is gratefully acknowledged.

\section{REFERENCES}

1. Wei F. and Pizhong Q. 2011. "Vibration-based Damage Identification Methods: A Review and Comparative Study," Structural Health Monitoring, 10(1):83-111.

2. Peeters, B. and G. D. Roeck. 1999. "Reference-based stochastic subspace identification for output-only modal analysis," Mechanical Systems and Signal Processing, 13(6):855-878.

3. Basseville, M., L. Mevel, and M. Goursat. 2004. "Statistical model-based damage detection and localization: Subspace-based residuals and damage-to-noise sensitivity ratios," Journal of Sound and Vibration, 275(3-5):769-794.

4. Döhler, M., L. Mevel, and F. Hille. 2014. "Subspace-based damage detection under changes in the ambient excitation statistics," Mechanical Systems and Signal Processing, 45(1):207224.

5. Döhler, M., L. Mevel, and Q. Zhang. 2016. "Fault detection, isolation and quantification from Gaussian residuals with application to structural damage diagnosis," Annual Reviews in Control, 42:244-256.

6. Döhler, M., F. Hille, L. Mevel, and W. Rücker. 2014. "Structural health monitoring with statistical methods during progressive damage test of S101 Bridge," Engineering Structures, 69:183-193.

7. Döhler, M. and L. Mevel. 2013. "Efficient multi-order uncertainty computation for stochastic subspace identification," Mechanical Systems and Signal Processing, 38(2):346-366.

8. Basseville, M., M. Abdelghani, and A. Benveniste. 2000. "Subspace-based fault detection algorithms for vibration monitoring," Automatica, 36(1):101-109.

9. Döhler, M. and F. Hille. 2014. "Subspace-Based Damage Detection on Steel Frame Structure Under Changing Excitation," in A. Wicks, ed., Structural Health Monitoring, Volume 5, Springer International Publishing, Cham, Conference Proceedings of the Society for Experimental Mechanics Series, pp. 167-174.

10. Benveniste, A. and B. Delyon. 2000. "Using local tests to estimate convergence rates for identification," in Proc. 39th IEEE Conference on Decision and Control, pp. 1985-1990.

11. Liu, J., X. Liu, and X. Ma. 2008. "First-Order Perturbation Analysis of Singular Vectors in Singular Value Decomposition," IEEE Transactions on Signal Processing, 56(7):30443049 . 